\title{
Mosquitoes score in chemical war
}

\section{Growing resistance is threatening global malaria-control efforts.}

\section{BY DECLAN BUTLER}

$\mathrm{K}$ ey weapons in the fight against malaria, pyrethroid insecticides, are losing their edge. Over the past decade, billions of dollars have been spent on distributing longlasting pyrethroid-treated bed nets and on indoor spraying. Focused in Africa, where most malaria deaths occur, these efforts have greatly reduced the disease's toll. But they have also created intense selection pressure for mosquitoes to develop resistance.

"Data are coming in thick and fast indicating increasing levels of resistance, and also of resistance in new places," says Jo Lines, an entomological epidemiologist and head of vector control at the Global Malaria Programme of the World Health Organization (WHO) in Geneva, Switzerland. The WHO now intends to launch a global strategy to tackle the problem by the end of the year.

Pyrethroids are the mainstay of malaria control because they are safe, cheap, effective and long-lasting. Alternatives such as organophosphates and carbamates are available for indoor spraying, although they cost more and are less effective. But pyrethroids are the only insecticides approved by the WHO for use in bed nets. "We have lots of our eggs in the pyrethroid basket," says Robert Newman, director of the Global Malaria Programme.

The international community has been slow to respond to the threat despite warnings, says Janet Hemingway, director of the Liverpool School of Tropical Medicine, UK, and chief executive of the non-profit Innovative Vector Control Consortium, a public-private venture set up in 2005 to develop new insecticides and monitoring tools. "A number of us had been banging the drums, saying: 'As soon as you scale up you are going to get resistance." But
Lines says that the malaria-control community felt too many lives were at stake to let the threat of resistance stand in the way of massively scaling up the bed-net and spraying campaigns.

Teasing out the impact of resistance on the success of malaria-control interventions is difficult because so many other factors influence their outcome. More systematic and more sophisticated monitoring of resistance is also vital, says Lines. The best surveillance data

increased levels of mosquito enzymes that can destroy pyrethroids before they reach their target, require more complex tests to detect $(\mathrm{H}$. Ranson et al. Trends Parasitol. 27,91-98; 2011).

But uncertainties about the extent of resistance or its impact are "no excuse for inaction", says Newman, arguing that the proposed WHO strategy needs to be urgently implemented, and also rolled out preemptively in places where resistance has yet to be detected. The WHO's plan will recommend, for example, that control programmes rotate insecticides sprayed indoors, using pyrethroids one year and a different class the next. This would be more costly and less effective than relying only on pyrethroids, however, so control programmes may be reluctant to adopt this measure.

Lines says that new combinations of insecticides also need to be developed, so that mosquitoes resistant to one would be killed by the other. In areas where pyrethroid bed nets are used, a different class of insecticides should be used for wall spraying, he adds.

Ultimately, entirely new classes of insecticides - particularly those that can be applied to bed nets are needed to alleviate the dependence of malaria-control efforts on pyrethroids. For indoor spraying, some longer-lasting and more costeffective non-pyrethroid insecticides should be available by next

(see 'Resistance on the rise'), although useful, do not give a complete picture of where resistance is emerging and how prevalent it is, he says. Malaria-control programmes often lack insectresistance monitoring, and detection of all forms of resistance is not easy. Quick, cheap tests can pick out gene mutations that help the mosquitoes' nerve cells withstand pyrethroid attack. But other forms of resistance, which depend on year, Hemingway says, although developing wholly new classes will take five to seven years. Repurposed agricultural insecticides might also act as a stopgap were resistance to pyrethroids to develop rapidly. Research targeting mosquito control is "grossly underfunded" compared with that on malaria drugs and vaccines, she adds, which is why control efforts have had so few options to call on.
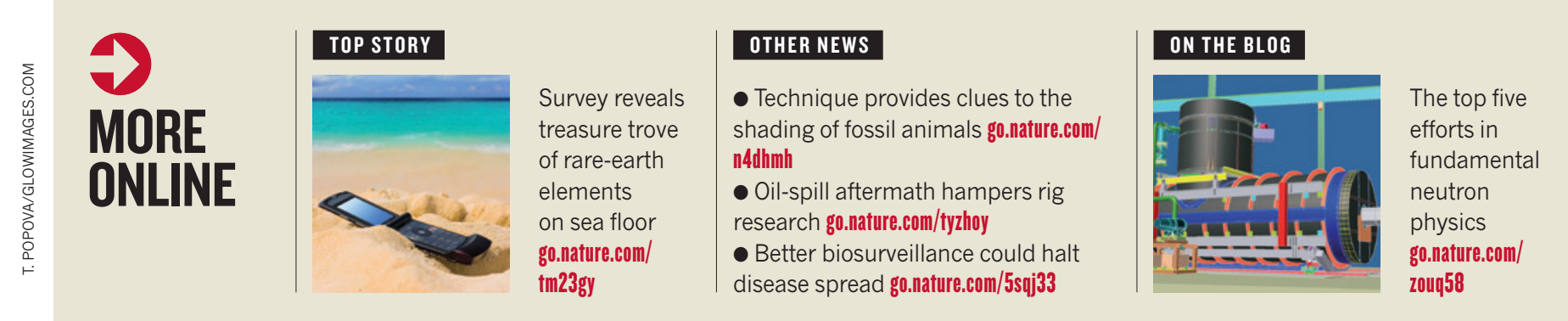